\title{
Arrhythmia Detection from ECG based Heartbeat Classification using Deep Learning Networks
}

\author{
Boyina Nagaraju \\ Student, M.Tech, CSE Dept \\ Gudlavalleru Engineering College \\ Gudlavalleru, India
}

\author{
Kalyanapu Srinivas \\ Assoc. Professor, CSE Dept \\ Gudlavalleru Engineering College \\ Gudlavalleru, India
}

\begin{abstract}
-ABSTRACT
Grouping is a standout amongst the most mainstream points in human services and bioinformatics, particularly in connection to arrhythmia location. Arrhythmias are abnormalities in the rate or musicality of the heartbeat which, now and again, may happen sporadically in a subject's day by day life. To catch these rare occasions, a Holter gadget is normally utilized to record long haul ECG information. Along these lines, the programmed acknowledgment of irregular pulses from a lot of ECG information is a significant and fundamental assignment. Over the most recent two decades, countless have been proposed to address the issue of ECG beat order. In the meantime, profound learning has progressed quickly since the mid 2000s and now shows a best in class execution in different fields. In this paper, we propose a novel profound learning approach for ECG beat grouping. We have led the tests on the outstanding MIT\{BIH Arrhythmia Database, and contrasted our outcomes and the logical writing. The last outcomes demonstrate that our model isn't just more productive than the best in class as far as exactness, yet in addition aggressive as far as affectability and particularity.
\end{abstract}

\section{Keywords}

Deep Learning, ECG characterization, Heartbeat grouping, arrhythmia discovery.

\section{INTRODUCTION}

The electrocardiogram (ECG) is a non-intrusive, reasonable and settled symptomatic apparatus broadly utilized in a few applications. It speaks to the progressions of the electrical movement of the heart after some time and contains basic 5 physiological data that is generally used to break down heart work. The heart electric field is recorded by methods for anodes on the body surface. It is estimated as a voltage (for example potential distinction) between two cathodes. A blend of two anodes that shapes a nonexistent line in the body along which the electric sign are estimated is known as a lead". 10 ECG sign are intermittent sign, since they are made out of a grouping of waves that rehash occasionally in time: a $\mathrm{P}$ wave, at that point $\mathrm{Q}, \mathrm{R}$ and $\mathrm{S}$ waves (which structure the $\mathrm{QRS}$ complex) and a $\mathrm{T}$ wave, as appeared in Figure 1. In all respects once in a while, can a $U$ wave likewise be distinguished. The most trademark some portion of an ECG signal is the QRS complex. In particular, the investigation of this complex relates to the 15 beat-to-beat grouping. An arrhythmia is an abnormality in the rate or musicality of the heartbeat. Amid an arrhythmia, the heart can thump excessively quick, excessively moderate, or with an unpredictable musicality. Most arrhythmias are innocuous, yet some can be not kidding or even perilous. Amid an arrhythmia, the heart will most likely be unable to siphon enough blood to the body. Such an absence of blood ow can harm the cerebrum, heart and different organs. At basic dimensions, heart arrhythmias can be sorted into two kinds, perilous and non-dangerous. Hazardous arrhythmias, for example, ventricular fibrillation and tachycardia, may trigger heart failure and abrupt 25 passing. Patients in these circumstances require crisis treatment. While non-dangerous arrhythmias may not prompt up and coming heart disappointment, opportune treatment is as yet expected to keep away from any further crumbling of the heart work.



Fig 1. A typical ECG trace, with a $P$ wave, $Q, R$ and $S$ waves (which form the QRS complex) and a $T$ wave, and the R-R interval labelled.

Sometimes, arrhythmias may just happen sporadically in a patient's everyday life. To catch these rare occasions, a Holter gadget is generally utilized to record long haul ECG information. In this way, the programmed acknowledgment of anomalous pulses from a lot of ECG information is a significant and basic undertaking. The primary kind of arrhythmia has been all around considered and some discovery calculations have been created and actualized in programmed outer defibrillators (AED). To perceive the second sort, distinguishing the ectopic beat is a basic advance. Moreover, for the investigation of long haul ECG chronicles, beat-by-beat manual examination is repetitive and tedious, particularly on account of bedside checking or wearable human services observing, where continuous conclusion is a troublesome assignment for junior specialists. Hence, clinicians more often than not utilize PC helped strategies to investigate and decipher the ECG signals. As additionally commented in on account of the fast development of novel detecting advances, the ECG information to be broke down isn't just gigantic in sum yet in addition complex in structure for conventional programming and equipment. Actually, a few reports have evaluated that advanced social insurance information from everywhere throughout the world totaled 500 petabytes (1015) in 2012. Also, it is required to increment to achieve exabytes in 2020. Subsequently, arrangements are required so as to oversee and examine such mind boggling, different, and colossal datasets with a sensible time multifaceted nature and capacity limit. Huge information expository, a prevalent term given to datasets that are 
substantial and complex, assumes an essential job in dealing with the gigantic measure of social insurance information and improving the nature of human services administrations offered to patients. In this specific situation, one of the difficulties lies in the characterization of this information, which depends on viably circulated preparing stages and propelled information mining and AI systems.

In this way, a profound learning method is acquainted in this work with address the difficulties looked by group the ECG beats. As of late, profound learning strategies have been utilized by numerous organizations, including dobe, Apple, Baidu, Facebook, Google, IBM, Microsoft, NEC, Netix, and NVIDIA, and in a vast arrangement of utilization areas, as in. In this paper, we present a novel profound learning approach for ECG beat order. The methodology has been created by utilizing the Tensor Flow system, the profound taking in library from Google, in the Python programming language. We have directed analyses on the outstanding MIT\{BIH Arrhythmia Database, and contrasted our outcomes and the logical writing. The last outcomes demonstrate that our model isn't just more proficient than the best in class as far as exactness, yet additionally aggressive as far as affectability and particularity. In the accompanying area, Section 2, we report an outline of the current programmed heartbeat order procedures and calculations, and remark on their highlights and attributes.we report an overview of the existing automatic heartbeat classification techniques and algorithms, and comment on their features and characteristics. The ECG-based heartbeat classification model is presented with a detailed description of the MIT-BIH Arrhythmia Database (MITBIH-AR) provided in the Details about the signal processing used to create the new dataset are while a description of its characteristics . The proposed Deep Neural Network (DNN)for the heartbeat classification is described and its performance in comparison with other well-known classifiersin terms of accuracy, sensitivity and specificity.

\section{RELATED WORK}

Electrocardiogram (ECG) is a non-intrusive, cheap and entrenched indicative apparatus broadly utilized in a few applications. It speaks to the progressions of the electrical action of the heart after some time and contains fundamental physiological data that is generally used to break down heart work. Highlight choice, otherwise called variable choice, trait choice or variable subset determination, is the way toward choosing a subset of pertinent highlights for use in model development. The focal suspicion when utilizing a component choice strategy is that the information contains numerous excess or insignificant highlights.

Redundant highlights are those which give no more data than the as of now chosen highlights,

Irrelevant highlights give no valuable data in any specific situation.

Highlight determination is likewise valuable as a feature of the information investigation process, as shows which highlights are significant for forecast, and how these highlights are connected. Order is a standout amongst the most well known points in medicinal services and bioinformatics, particularly for arrhythmia discovery. Arrhythmias are abnormalities in the rate or beat of the heartbeat which, now and again, arrhythmias may happen sporadically in a subject's day by day life. To catch these rare occasions, 430 a Holter gadget is typically utilized to record long haul ECG information. Accordingly, the programmed acknowledgment of strange pulses from a lot of ECG information is a significant and basic assignment. Be that as it may, the extent of the information is voluminous(BigData) to start any examination to derive blockage estimations. Numerous information mining techniques and classifier, for example, SVM, Bayesian endeavored to process information however endured seriously regarding powerlessness to prepare these classifiers over BigData Leading to Out Of Memory Exceptions and henceforth prompting mistaken arrhythmia location. So we need a superior framework to improve these expectation models that can procedure these vast volumes of ECG information to find strange ecg estimations better. Thinking about every one of the prospects and worries of all the component determination and grouping strategies, there is positively a requirement for a superior methodology that has not too bad exactness, all inclusive statement and low computational intricacy for dealing with enormous ECG information.

Artificial Neural Networks (ANN) are computational calculations enlivened by networks of organic neurons to take care of expectation issues in PC vision, common language handling and medication disclosure, etc.Smaller impression models may likewise acquire focal points requiring less preparing information, and in being conceivably increasingly versatile to changing target areas. In this paper, we present an exhaustive investigation of little impression ECG arrangement models utilizing deep neural networks (DNNs). A DNN is a specific fake neural system with multiple layers, which naturally intertwines include extraction and order into a sign learning body and legitimately develops a basic leadership work. By and large, a DNN comprises of an info layer for the crude descriptors $\mathrm{X}, \mathrm{L}$ concealed layers, and a yield layer for the forecast. Deep Neural Network (DNN) for the programmed characterization of irregular ECG beats. The proposed DNN was tried upon an engineered ECG dataset and the effectiveness of our outcomes is featured in our exhibit.

\section{PROPOSED METHODOLOGY}

\subsection{Database mit $\{$ bih arrhythmia database\}}

The MIT-BIH arrhythmia database was utilized in this examination. It is an openly available database which has been generally used to assess execution of various ECG-based heartbeat grouping calculations. The MIT-BIH arrhythmia database involves 48 records of ECG gathered from 47 subjects (record 201 and 202 originated from a similar male subject) and each record contains 30 minutes in length twochannel ECG signals which were separated utilizing a 0.1 to $100 \mathrm{~Hz}$ bandpass channel and digitized at $360 \mathrm{~Hz}$. Each beat has been named by two cardiologists autonomously and the planning of the $\mathrm{R}$ crests (or the nearby extremum) for each beat is additionally given. The names have been ceaselessly refreshed in the previous couple of decades. The information were dealt with as indicated by AAMI ECAR-1987 prescribed practice for assessment of our order calculation. Similar information taking care of technique was utilized in and and our outcomes can be contrasted with them straightforwardly. Four records (\#102,\#104, \#107 and \#217) were prohibited in light of the fact that they contain paced pulses. In light of the qualities and side effects of the subjects, the 44 records utilized can be isolated into two gatherings. The principal gathering (incorporates 20 records with marks \# beginning with 1) fill in as the delegate test of an assortment of waveforms and curios that an arrhythmia locator may experience in routine clinical use. The rest of the 24 (records with names \# beginning with 2) incorporate complex ventricular, junctional and supraventricular arrhythmias and conduction variations from the norm. Multifaceted nature in 
beat, QRS morphology variety or sign quality in a portion of these records will exhibit critical test to arrhythmia indicators. As per the AAMI suggestion every ECG beat can be named $\mathrm{N}$ (beats starting in the sinus mode), S (supraventricular ectopic beats), $\mathrm{V}$ (ventricular ectopic beats), $\mathrm{F}$ (combination beats) or Q (unclassifiable beats). To prepare a subject explicit classifier, the preparation information comprised of two sections, a typical part and a subject explicit part. The regular piece of preparing information was chosen from the principal gathering (record \# began with 1) and is utilized for all testing subjects (second record gathering, record \# began with 2). Following the AAMI prescribed practice, at most 5 minutes of chronicles from a subject were utilized for classifier preparing reason. So the subject explicit piece of preparing information incorporated the pulses from the initial 5 minutes of the ECG recording of each testing subject. The rest of the 25 minutes of the record was utilized for testing.Each ECG record of the MIT-BIH Arrhythmia Database includes two leads originating from different electrodes.
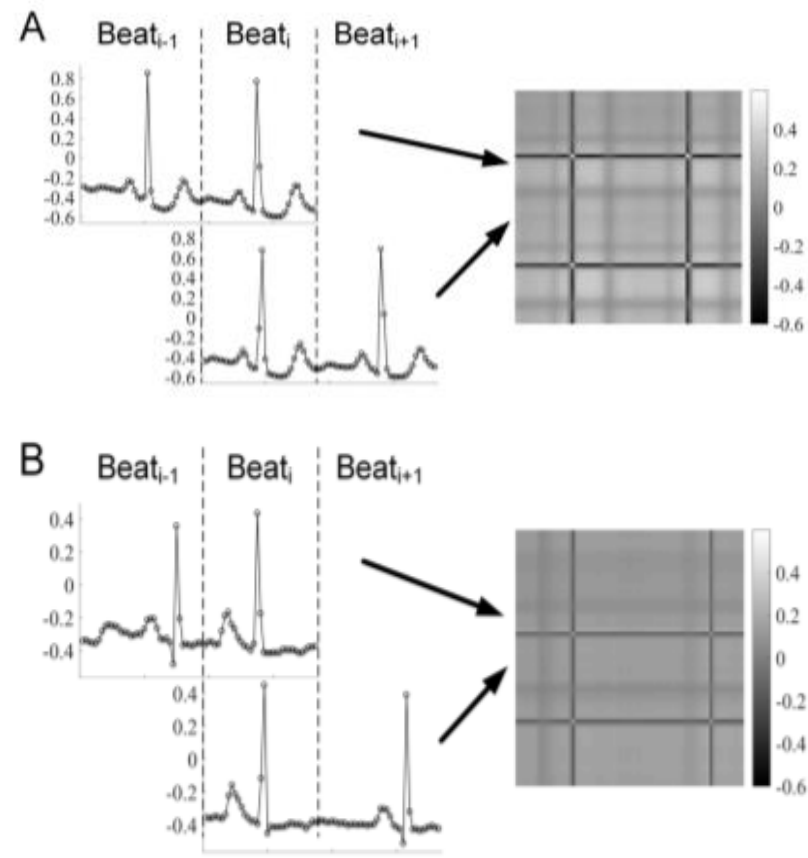

Fig 2. Dual-beat coupling matrix computed from two pairs of adjacent beats. A. Coupling matrix of beats originating from the sinus mode. B. Coupling matrix from a supraventricular ectopic beat.

\subsection{Ecg signal preprocessing}

In this investigation, we utilized the adjusted lead II channel just from the database. Programmed ECG grouping is especially valuable for versatile or wearable gadget and it is normal that few channel number (even single channel) would be found in these gadgets. Thus, we built up our calculation to deal with little channel number of ECG. The planning of every heartbeat has been marked for the comparing $\mathrm{R}$ crest in the database. Thus, we can legitimately acquire the R-R interims for each beat in our division. In any case, various hearty strategies have just been accessible for $\mathrm{R}$ crest identification and calculation for this is past the extent of current investigation.Starting from the MIT-BIH Arrhythmia Database we have created a new dataset by applying to the ECG signals the following processing steps.

Denoising:to eliminate possible power line interference and baseline wanderings caused by respiration or patient movements that can create problems in the detection of the ECG peaks.

Peak Detection: to determine the positions of all peaks of interest found in the ECG signal. The p-wave and $t$ wavepositions are useful for the signal segmentation. Instead, the r-peak detection is essential for the temporal features extraction.

Signal Segmentation: to divide the ECG signal into single beats that will be classified as normal or abnormal.

Temporal features extraction: to extract information about the ECG signal trend or, more precisely, about the variability of the signal.

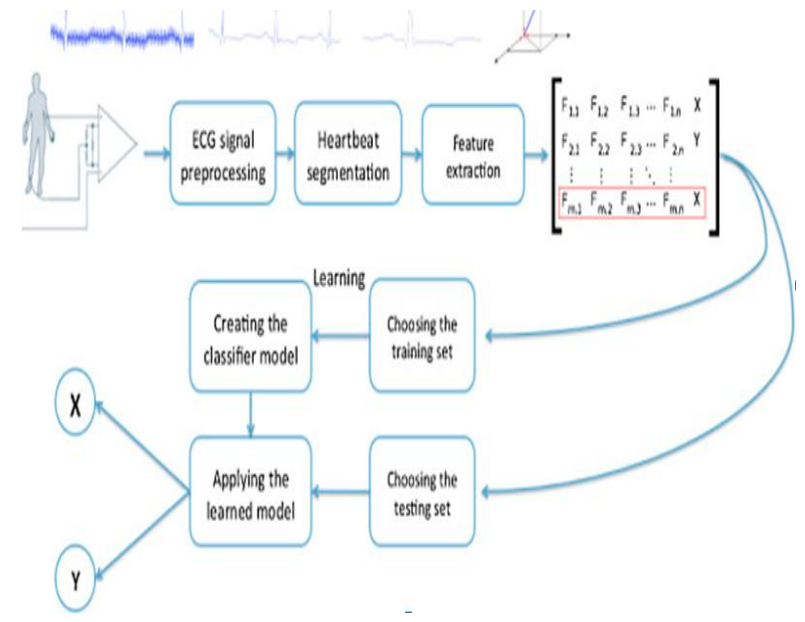

Fig 3:Overview of all processing steps.

\subsubsection{Heartbeat segmentation}

The morphology of every heartbeat is basic for order of arrhythmia. A few examinations sectioned the ECG signals into equivalent length at preprocessing. Notwithstanding, the heartbeat rates may shift fundamentally among various subjects and after some time, and henceforth each beat is of various length. Therefore, the heartbeat rate, and henceforth the beat length, ought to be viewed as powerfully after some time and explicitly for each subject. The beat was portioned with the end goal that it was based on the $\mathrm{R}$ crest. For each record in the main gathering (the basic part), the division length is processed as the normal $R-R$ interim over $\pm T$ sec from the present beat. Consequently, the length for each beat is variable. In this investigation, we picked $T=10 \mathrm{sec}$ with the end goal that the normal R-R interim is determined over $20 \mathrm{sec}$. For each record in the second gathering (the subject explicit part, both preparing and testing beats), the beats are fragmented utilizing the R-R interim arrived at the midpoint of over the initial 5 minutes of the record. In that capacity, every one of the beats in the second gathering for each subject have equivalent length. Utilizing normal R-R interim over each 20 sec yielded comparative outcomes in our examination.

\subsubsection{Segment length scaling}

So as to bring together the diverse section length because of variety in heartbeat rate for contributing into the classifier, the portions are first scaled into a similar length. On the off chance that the length (in test number) of an extricated beat is $\mathrm{Ni}$, we will initially up-test it, by interjection, by a factor equivalents to the info estimate, $\mathrm{M}$, intended for the CNN. Henceforth, the length of the beat moves toward becoming $\mathrm{Ni} \times \mathrm{M}$. At that point, the mean qualities for each Ni tests are determined to such an extent that in the long run, we can acquire a section of length $\mathrm{M}$ for beat with any unique length 
of $\mathrm{Ni}$.

\subsection{Inputs for classifier}

The double beat coupling lattice, which incorporates both beat waveform and beat-to-beat relationship was utilized as contribution to this examination. Heartbeat arrhythmia can be dissected by single beat morphology, yet in addition beat-tothump connection. Consequently, we considered a progression of three neighboring beats in defining the contribution for the classifier. Two sets of heartbeat are separated after a similar division guideline as portrayed previously. The coupling lattices incorporate both morphological and musical data of the ECG into a solitary information. For example, in Fig. 1, the four high force focuses (white) relate to the four $\mathrm{R}$ pinnacles of the two sets of heartbeat in concern. The force mirrors the overall abundancy of R tops in these the beats, while the general position of these focuses mirrors the musicality of this section of ECG. Fig. 1A demonstrates a portion of ECG started from sinus mode, while Fig. 1B demonstrates a section around a supraventricular ectopic beat. In Fig. 1A, the four white focuses disseminate all the more equitably over the grid and it compares to a customary beat. Different pieces of the coupling framework give additional data about the sections, for example, the morphology and beat of $\mathrm{P}, \mathrm{Q}, \mathrm{S}$ and $\mathrm{T}$ waves.

\section{WORK OVERVIEW:}

We propose a far reaching investigation of much littler impression enormous information handling models utilizing parkway deep neural networks (HDNNs). HDNNs are multilayer networks which have easy route associations between shrouded layers. Contrasted with ordinary multi-layer networks with skip associations, HDNNs are moreover outfitted with two entryway capacities - change and convey gates- which control and encourage the data stream all through the entire system. Specifically, the change entryway scales the yield of a shrouded layer, and the help door is utilized to go through a layer input straightforwardly after component astute rescaling. These entryway capacities are integral to preparing deep networks and to accelerating combination. We demonstrate that for Big Data ECG Processing, arrhythmia discovery and precision can be held by expanding the profundity of the system, while the quantity of shrouded units in each concealed layer can be fundamentally diminished. Subsequently, HDNNs are a lot more slender and deeper with numerous less model parameters. In addition, as opposed to preparing normal multi-layer networks of a similar profundity and width, which commonly requires cautious pre preparing, we show that HDNNs might be prepared utilizing standard stochastic slope plummet. Our key perception is that the door capacities can control the conduct of all the shrouded layers, and they are strong to over fitting, in this manner creating an improved exactness of extensive estimated HDNN acoustic wave models.

\section{ASSESSMENT INDICATORS}

To evaluate the performance of the proposed classifier, we used four statistical indicators in this study, which have been commonly used in previous work. They are classification accuracy (Acc), sensitivity (Sen), specificity (Spe) and positive predictive rate (Ppr). The classification Acc measures the overall performance of the proposed method on all valid heartbeats. However, as the number of different types of beats varies, Sen, Spe and Ppr are less biased in assessing the classifier performance. The four statistical indicators can be calculated as follow

$$
\begin{gathered}
\mathrm{Acc}=\frac{\mathrm{TP}+\mathrm{TN}}{\mathrm{TP}+\mathrm{TN}+\mathrm{FP}+\mathrm{FN}} \\
\text { Sen }=\frac{T P}{T P+F N}
\end{gathered}
$$

True Positive (TP) is the number of abnormal beats correctly classified;

True Negative (TN) is the number of normal beats correctly classified;

False Positive (FP) is the number of normal beats incorrectly classified as abnormal;

False Negative (FN) is the number of abnormal beats incorrectly classified as normal.

\section{RESULTS}

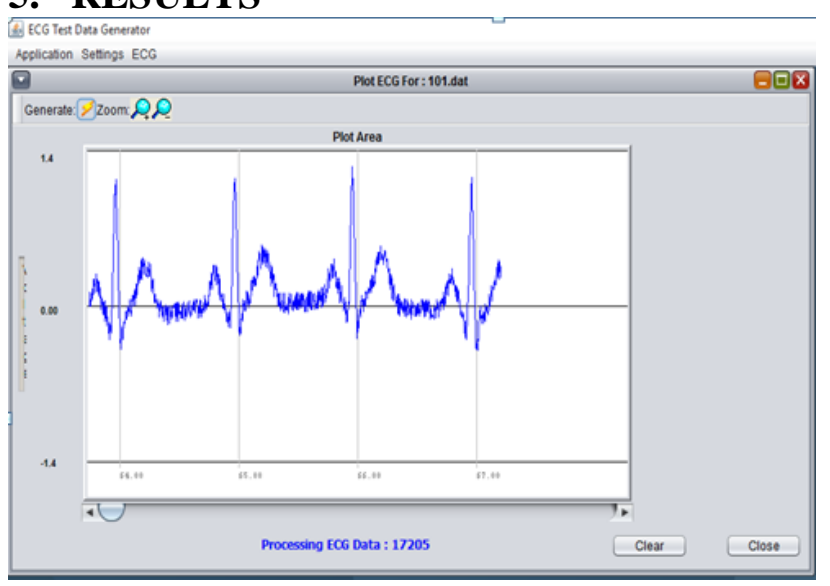

Fig 4: ECG 101 data

Figure 4 describes the duration time for processing ECG data. This figure output is based on deep neural network algorithm. This algorithm takes a lot of time for processing ECG data. So we need to do enhancement which further decreases the processing time.

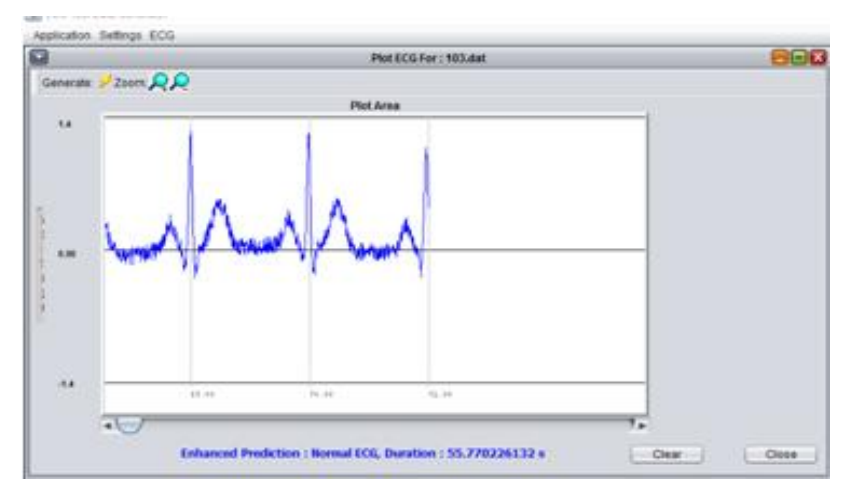

Fig 5: ECG 101 data(normal ECG)

Figure 5 describes the duration time for processing ECG data. This figure 5 output is based on High way deep neural network algorithm. By this algorithm the processing time can be reduced efficiently for 101 data. 


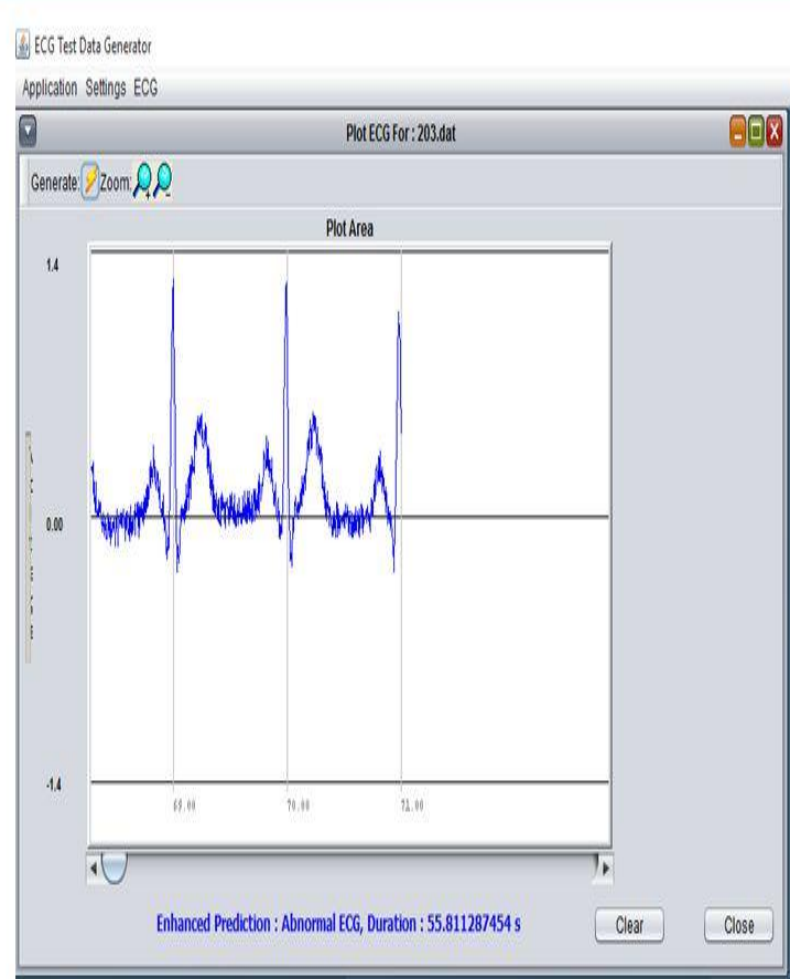

Fig 6: ECG 203 data(abnormal ECG)

Figure 6 describes the duration time for processing ECG 202 data. This figure 6 output is based on High way deep neural network algorithm. By this algorithm the processing time can be reduced efficiently for 203 data(abnormal ECG).

\begin{tabular}{|c|c|c|c|c|c|c|c|}
\hline (19+0; & & 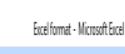 & & & & & $0 x$ \\
\hline (3) & 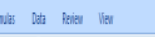 & & & & & & 8.9 \\
\hline 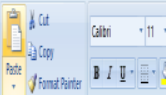 & 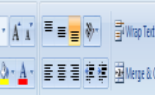 & athe & 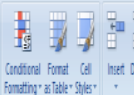 & I I & 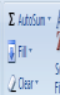 & & \\
\hline comed & Hypert & latse & 据 & ato & tit & & \\
\hline 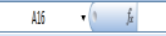 & & & & & & & \\
\hline$A$ & $B$ & c & 0 & & & $F$ & G \\
\hline 1 File Name & System & Dectiton Rate & False Postivies & & & & \\
\hline 2 205.0at & Existing & 80.3 & 37 & 0 & & & \\
\hline 3 101.0at & Existing & 80.4 & 45 & 0 & & & \\
\hline 4 100.Dat & Existing & 80.4 & 47 & 0 & & & \\
\hline 5 104.Dat & Existing & 80.4 & & 1 & & & \\
\hline 6 201.Dat & Proposed & 82.6 & & 0 & & & \\
\hline 7 102.Dat & Proposed & 83.0 & & 0 & & & \\
\hline 8 203.Dat & Proposed & 82.9 & & 0 & & & \\
\hline 9 103.Dat & Enhanced & 55.7 & & 0 & & & \\
\hline 10 207.Dat & Enhanced & 55.8 & & 0 & & & \\
\hline 11 & & & & & & & \\
\hline 12 & & & & & & & \\
\hline$\frac{13}{x+1+1} \operatorname{sint1} 5$ & & & & & & & \\
\hline
\end{tabular}

\section{Dectiton Rate}

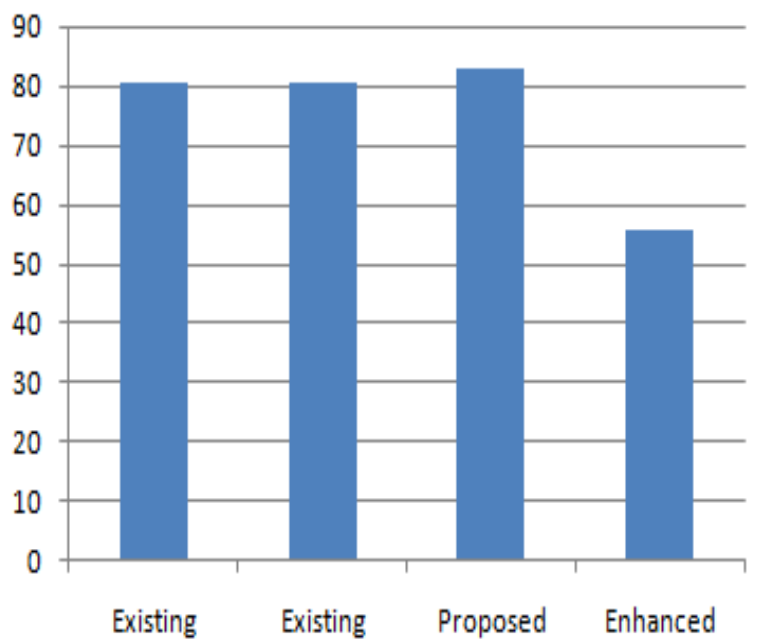

Classification of the result processing Accuracy and less time series of Dection Rate.

\section{CONCLUSION}

Classification is a standout amongst the most mainstream points in human services and bioinformatics, particularly for arrhythmia discovery. Arrhythmias are abnormalities in the rate or mood of the heartbeat which, now and again, arrhythmias may happen sporadically in a subject's day by day life. To catch these rare occasions, a Holter gadget is generally utilized to record long haul ECG information. In this manner, the programmed acknowledgment of strange pulses from a lot of ECG information is a significant and fundamental errand. In this paper, we have proposed a methodology dependent on a Deep Neural Network (DNN) for the programmed order of anomalous ECG beats, separated from ordinary ones. DNN has been created by utilizing the Tensor Flow structure, the deep taking in library from Google, and it is made out of just seven concealed layers, with 5,10 , $30,50,30,10$ and 5 neurons, separately. To exhibit the quality, as far as right arrangement, of the proposed DNN, we have completed an exact examination by considering eleven other surely understood classifiers on account of the utilization of the WEKA apparatus. The numerical outcomes have demonstrated the adequacy of the methodology, particularly as far as exactness. Our future research will include the utilization of other uninhibitedly accessible databases, as INCART and SVDB, so as to assess the accuracy of the methodology and of the proposed DNN, and furthermore so as to enhance the learning capacity of the neural system. Also, we are making arrangements for our way to deal with be inserted in a constant ECG observing framework and to be tried in a true circumstance by methods for a nearby co-activity with a particular emergency clinic division. Another fascinating issue worth examining is that identified with the association of doctors all the while, with reference to the explanations. Truth be told, as found in the depiction of the database, there are as of now such a large number of ECG beats which must be explained for this to be utilized in the preparation or testing period of the DNN. 


\section{REFERENCES}

[1] L. Carnevale, A. Celesti, M. Fazio, P. Bramanti, M. Villari, Heart disorder detection with menard algorithm on apache spark, in: EuropeanConference on ServiceOriented and Cloud Computing, Springer, 2017, pp.229.

[2] P. Melillo, R. Castaldo, G. Sannino, A. Orrico, G. De Pietro, L. Pecchia,Wearable technology and ecg processing for fall risk assessment, prevention 470 and detection, in: 37th Annual International Conference of the IEEE Engineering in Medicine and Biology Society (EMBC), 2015, pp. 7740.

[3] G. Sannino, G. De Pietro, An evolved ehealth monitoring system for anuclear medicine department, in: Developments in E-systems Engineering (DeSE), IEEE, 2011, pp. 3.

[4] P. Laguna, R. Jan_e, S. Olmos, N. V. Thakor, H. Rix, P. Caminal, Adaptiveestimation of qrs complex wave features of ecg signal by the hermitemodel,Medical and Biological Engineering and Computing 34 (1) (1996) pp. 58.

[5] A. Amann, R. Tratnig, K. Unterkoer, Detecting ventricular _brillation by time-delay methods, IEEE Transactions on Biomedical Engineering 54 (1) (2007) pp. 174.

[6] U. Irusta, J. Ruiz, S. R. de Gauna, T. Eftest_1, J. KramerJohansen, Aleast mean-square _lter for the estimation of the cardiopulmonary resuscitation artifact based on the frequency of the compressions, IEEE Transactions 485 on Biomedical Engineering 56 (4) (2009) pp. 1052.

[7] E. Aramendi, U. Irusta, E. Pastor, A. Bodegas, F. Benito, Ecgspectraland morphological parameters reviewed and updated to detect adult andpaediatric life-threatening arrhythmia, Physiological measurement 31 (6) (2010) p. 749

[8] R. Fang, S. Pouyanfar, Y. Yang, S.-C. Chen, S. Iyengar, Computationalhealth informatics in the big data age: A survey, ACM Computing Surveys(CSUR) 49 (1) (2016) p. 12.

[9] J. Sun, C. K. Reddy, Big data analytics for healthcare, in: Proceedings ofthe 19th ACM SIGKDD international conference on Knowledge discoveryand data mining, ACM, 2013, pp. $1525\{1525$.

[10] I. Goodfellow, Y. Bengio, A. Courville, Y. Bengio, Deep learning, Vol. 1,MIT press Cambridge, 2016 http://www.deeplearningbook.org.

[11] F. Celesti, A. Celesti, L. Carnevale, A. Galletta, S. Campo, A. Romano,P. Bramanti, M. Villari, Big data analytics in genomics: The point on deeplearning solutions, in: IEEE Symposium on Computers and Communications (ISCC), IEEE, 2017, pp. 306.

[12] O. E. Isa_ade, O. E. Isa_ade, A. B. Bagula, Data mining trends and applications in criminal science and investigations, 1st Edition, IGI Global,Hershey, PA, USA, 2016, pp. 1. 